the continuity and regularity of the tonic discharges was lost and only short trains at low frequency (5-10 sec. $\left.{ }^{-1}\right)$ remained. At this stage the phasic response is reduced to a few impulses (Fig. 4), and the range of amplitudes becomes reduced, showing that there are fewer active fibres.

The preparations usually survived for many hours. The simplicity of the experimental procedure and the regularity of the responses make this preparation a very convenient one for physiological and toxicol. ogical investigations.

\section{G. BECHT}

Laboratory for Research on Insecticides, Vondellaan 6, Utrecht.

'Wiersma, C. A. G., Furshpan, E., and Florey, E., J. Exp. Biol., 30 136 (1953).

${ }^{2}$ Finlayson, L. H., and Lowenstein, O., Nature, 176, 1031 (1955)

s Pringle, J. W. S., J. Exp. Biol., 33, 658 (1956).

4 Wigglesworth, V. B., "Principles of Insect Physiology" (London, 1950).

${ }^{5}$ Hughes, G. H., Nature, 170, 531 (1952).

- Nijenhuis, E. D., and Dresden, D., Proc. Kon. Ned. Akad. Amsterdam, C, 55, 300 (1952); C, 58, 121 (1955).

? Roeder, K. D., and Weiant, E. A., Science, 103, 304 (1946)

\section{Direct Determination of the Active Phase of End-Plate Potential}

IT is well known that the duration of transmitter action in neuromuscular transmission is very brief, of the order of 4 msec., and prolonged decline of the end-plate potential is due to the slow dissipation of charge along and across the resting membran $\theta^{1-4}$. The time course of the active phase of the neuromuscular transmission has been calculated by some authors $\mathbf{1}^{\mathbf{1}, 3}$, but it has not yet been obtained experimentally. In the present investigation the time course of the active phase of the transmitter producing the end-plate potential was recorded by a refined voltage clamp method (the original method was described by Hodgkin et al. ${ }^{5}$ ).

Two microelectrodes filled with $3 M$ potassium chloride were inserted into the end-plate focus of the curarized frog sartorius muscle. One of these electrodes recorded the membrane potential, and the feed-back amplifier regulated the current entering the other electrode in such a way as to hold the membrane potential at the resting value during the neuromuscular transmission. The membrane potential and the current which flowed through the current electrode were recorded with a double-channel oscilloscope. The focus of the end-plate was carefully located with multiple re-insertion of the electrode, and the current electrode was inserted within $50 \mu$ of the recording electrode. In order to incresse the feedback factor and also to reduce the capacitative escape of the feed-back current, a low-resistance electrode of about $5-10 \mathrm{M} \Omega$ was used especially for the current electrode. Fig. 1a shows the end-plate potential without feed-back and Fig. $1 b$ the membrane potential clamped at the resting potential with negative feed-back and the feed-back current entering the current electrode during the neuromuscular trans. mission. The time course of the feed-back current represents the time course of the active phase, with out electrotonic dissipation of charge, which produces the end-plate potential.

The general time course of the current was very similar to that of the active phase obtained by cal-
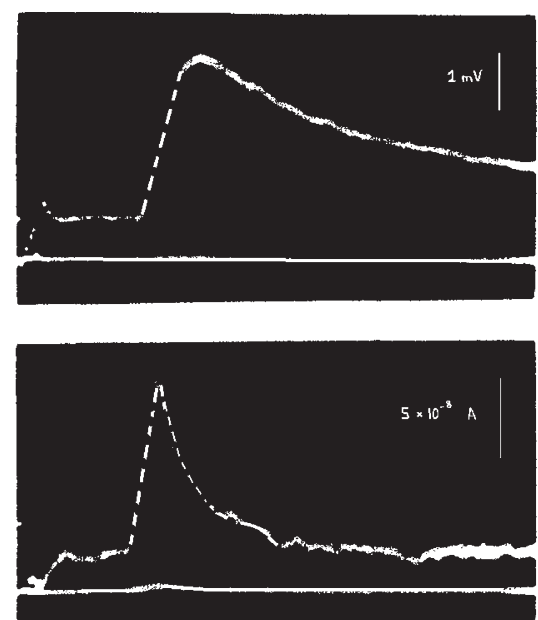

(b)

Fig. 1. $a$, End-plate potential recorded intracellularly from a curarized frog muscle; $b$, upper record-feed-back current flowing through the current electrode, so as to clamp the membrane potential at the resting value; lower record-the mem brane potential held at the resting level with a voltage clamp method during the neuromuscular transmission. All records wer recorded from the same end-plate using the double-channelled oscilloscope

culation ${ }^{1,3}$. The duration of rising phase was about 0.5 msec., and the peak to half-decay time was $0.6-0.9$ msec., the total duration being 3-4 msec., at $18^{\circ} \mathrm{C}$. The time course was prolonged by lowering the temperature or eserinizing the preparation, as will be reported in a later communication.

NoRIKo TAKEUCHX

Akrra Takeuchi

Department of Physiology,

Faculty of Medicine,

Juntendo University, Tokyo.

${ }^{1}$ Eccles, J. C., Katz, B., and Kuffler, S. W., J. Neurophysiol., 4, 362 (1941).

Kuffler, S. W., J. Neurophysiol., 5, 309 (1942).

Katz, B., Proc. Roy. Soc., B, 135, 506 (1948).

- Fatt, P., and Katz, B., J. Physiol., 115, 320 (1951).

Hodgkin, A. L., Huxley, A. F., and Katz, B., J. Physiol., 116, 424

(1952).

\section{Abrasion of the Insect Cuticle by Aqueous Suspensions of Small Particles}

I HAVE recently had occasion to require a standard or repeatable degree of abrasion of the cuticle of an insect, the larva of Rhodnius ${ }^{1}$. It was observed that in regions where excreta containing crystals of uric acid had dried on the surface, the cuticle appeared to be more permeable, suggesting that the drying of suspensions containing solid particles might produce abrasion. This was tested by applying suitable suspensions in distilled water, allowing them to dry in air, removing the particles in a stream of water and then testing for abrasion by exposure to ammoniacal silver hydroxide ${ }^{2}$.

Process white (barium sulphate), 'Almicide' (fine crystalline aluminium hydroxide), 'Carborundum' 500 , and activated charcoal were used as test materials. In all cases there was extensive abrasion of the surface as revealed by silver staining (Fig. 1). This abrasion was most evident over the crests of the folds in the cuticle, although the particles were deposited also in the depressions. Abrasion was usually 